\title{
Monotone Triangles and 312 Pattern Avoidance
}

\author{
Arvind Ayyer ${ }^{1,2}$ Robert Cori ${ }^{3} \quad$ Dominique Gouyou-Beauchamps ${ }^{4}$ \\ ${ }^{1}$ Institut de Physique Théorique \\ 2 Department of Mathematics \\ IPht, CEA Saclay \\ and URA 2306, CNRS \\ University of California, Davis \\ Davis, CA 95616, U.S.A. \\ 91191 Gif-sur-Yvette Cedex, France \\ ayyer@math . ucdavis . edu

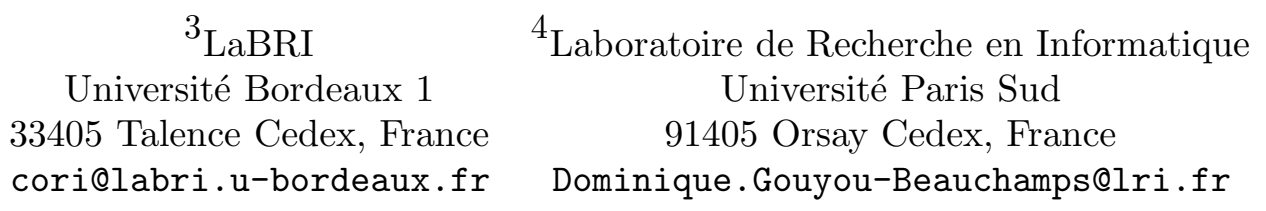 \\ Submitted: Jan 13, 2011; Accepted: Oct 25, 2011; Published: Nov 7, 2011 \\ Mathematics Subject Classification: 05A05, 05A10, 05A15, 05A16
}

\section{Dedicated to Doron Zeilberger on the occasion of his sixtieth birthday}

\begin{abstract}
We demonstrate a natural bijection between a subclass of alternating sign matrices (ASMs) defined by a condition on the corresponding monotone triangle which we call the gapless condition and a subclass of totally symmetric self-complementary plane partitions defined by a similar condition on the corresponding fundamental domains or Magog triangles. We prove that, when restricted to permutations, this class of ASMs reduces to 312-avoiding permutations. This leads us to generalize pattern avoidance on permutations to a family of words associated to ASMs, which we call Gog words. We translate the gapless condition on monotone triangles into a pattern avoidance-like condition on Gog words associated. We estimate the number of gapless monotone triangles using a bijection with $p$-branchings.
\end{abstract}

\section{Introduction}

The enumeration formula for ASM matrices was first proved by Doron Zeilberger [Zei96] by showing that monotone (or Gog) triangles naturally associated to them are equinumerous to Magog triangles associated to totally symmetric self-complementary plane partitions. Since the enumeration formula was known for these plane partitions this gave the 
result. The proof needed a long list of computations and since then, the construction of a bijection between Gog and Magog triangles has been and still is an open problem. We consider here a family of monotone triangles, which we call gapless, for which we show a simple bijection with a subclass of Magog triangles also satisfying a gapless condition.

It turns out that among monotone triangles associated to permutations, the gapless ones are exactly those corresponding to permutation avoiding the pattern 312 . This leads us to give a pattern avoidance-like characterization for ASMs whose monotone triangles are gapless. For this purpose we introduce a family of words associated to monotone triangles and generalize to these words the notion of avoidance of the pattern 312 . We note in passing that this notion of pattern avoidance for ASMs is different from that considered by Johansson and Linusson [JL07.

One of the main aims of this article is to build on the relationship between alternating sign matrices and totally symmetric self-complementary plane partitions first considered by Mills, Robbins and Rumsey MRR82. The other aim is to extend the notion of pattern-avoidance to other combinatorial structures related to permutations. One of the first works in this direction known to us is that of Hal Canary Can08 who established a relation between tilings of an Aztec diamond and alternating sign matrices using Baxter permutations.

We are also interested in asymptotic estimates for the number of gapless monotone triangles and for comparison, for the number of alternating sign matrices. We note that the general theory for asymptotic estimates of products of gamma functions like these is known (see, for example, Tem96]). Since we are mainly interested in the leading growth term for both quantities, we compute them from scratch in the appendix.

The organization of the paper is as follows. We first remind the reader of monotone (or Gog) triangles in Section 2, We then define the class of gapless monotone triangles for which we investigate their combinatorial structure and give an algorithm to build them in Sections 3 and 4 . We analyze a subset of these gapless triangles of rectangular shape in Section 5. A natural simple bijection from gapless monotone triangles to gapless Magog triangles is then shown in Section 6. We next proceed to show the relationship between monotone triangles and 312 patterns for permutations in Section 7 . This will turn out to be the simple case for the main theorem about 312 subpatterns in Gog words, which we define in Section 8. The proof of the pattern-avoidance theorem is then given in Section 9. We also give asymptotic estimates of the number of gapless monotone triangles in Appendix $\mathrm{A}$ and, for completeness, leading asymptotics of the number of alternating sign matrices in Appendix B. We summarize our results and give directions for future work in Section 10.

\section{Monotone triangles}

Definition 1. A monotone triangle or strict Gelfand pattern or Gog triangle of size $n$ is an array $\pi=\left(a_{i j}\right)$ of positive integers defined for $n \geq i \geq j \geq 1$ that is written in the 
form

$$
\begin{array}{cccc}
a_{1,1} & & & \\
a_{2,1} & a_{2,2} & & \\
\vdots & \vdots & \ddots & \\
a_{n, 1} & a_{n, 2} & \cdots & a_{n, n},
\end{array}
$$

where

1. $a_{i, j}<a_{i, j+1}, a_{i, j} \leq a_{i-1, j}$ and $a_{i, j} \leq a_{i+1, j+1}$ whenever both sides are defined. That is to say, numbers are increasing along rows, decreasing weakly along columns, and increasing weakly along diagonals.

2. $a_{n, j}=j$.

We note that this is close to the original convention in MRR83 for writing the triangles. However, it is different from the convention in [Zei96]. Monotone triangles are in natural bijection with alternating sign matrices MRR83. Since permutation matrices are a class of ASMs, one can express each permutation as a monotone triangle. The way to do this is to write the $i$ th row as an ascending list of the first $i$ elements in the permutation. For example, the permutation 4312 can be written as

$\begin{array}{llll}4 & & & \\ 3 & 4 & & \\ 1 & 3 & 4 & \\ 1 & 2 & 3 & 4,\end{array}$

We now define a special subset of monotone triangles; those with a gap.

Definition 2. A monotone triangle a contains a gap at position $(i, j)$ if $a_{i, j}-a_{i+1, j}>1$. A gapless monotone triangle is a monotone triangle which contains no gaps.

In the above example (2.2), the monotone triangle contains a gap at position $(2,1)$. The monotone triangle corresponding to the identity permutation is an extreme example of a gapless one. The column-wise difference in that case is zero everywhere.

In the definition of a monotone triangle, condition (1) consists in the satisfaction of three inequalities, notice that the third one can be omitted if the triangle is assumed to be gapless, since for integers the relation:

$$
a_{i, j}<a_{i, j+1} \leq a_{i+1, j+1}+1
$$

implies $a_{i, j} \leq a_{i+1, j+1}$.

More precisely we have:

Remark 1. A triangle $\left(a_{i j}\right)$ of positive integers, (where $n \geq i \geq j \geq 1$ ) is a gapless monotone triangle if the following conditions are satisfied:

(0) $\quad a_{i, j} \leq a_{i-1, j} \leq a_{i, j}+1$

$(1)^{\prime} \quad a_{i, j}<a_{i, j+1}$

(2) $\quad a_{n, j}=j$ 


\section{Shapes of gapless monotone triangles}

To any gapless monotone triangle $\left(a_{i, j}\right)$ of size $n$ we associate a shape, that is an array $\left(s_{i, j}\right)$ of size $n-1$ containing entries 0 and 1 and defined by

$$
s_{i, j}=a_{i, j}-a_{i+1, j},
$$

for $1 \leq j \leq i<n$. For instance the shape of

$\begin{array}{llll}3 & & & \\ 2 & 4 & & \\ 2 & 3 & 4 & \\ 1 & 2 & 3 & 4,\end{array}$

is

$\begin{array}{lll}1 & & \\ 0 & 1 & \\ 1 & 1 & 1\end{array}$

We remark that any triangle $\left(s_{i, j}\right)$ with 0,1 entries is not necessarily the shape of a gapless monotone triangle. A simple characterization can be given by examining the partial sums of two consecutive columns. To any triangle $\left(s_{i, j}\right)$ of size $n-1$ with 0,1 entries we associate $n-1$ words $f^{(1)}, f^{(2)}, \cdots, f^{(n-1)}$, on the alphabet $\{0,1\}$, representing its columns. More precisely the word $f^{(j)}$ is of length $j$ and given by:

$$
f^{(j)}=s_{n-1, n-j} \cdots s_{n-j, n-j} .
$$

Proposition 1. Two words $g$ and $h$ on the alphabet $\{0,1\}$ correspond to two consecutive columns in the shape of a gapless monotone triangle if and only their lengths $|g|,|h|$ satisfy $|h|-|g|=1$ and for any $i \leq|g|$ we have

$$
\sum_{k=1}^{i} g_{k} \geq \sum_{k=1}^{i} h_{k}
$$

Moreover a sequence of words $f^{(1)}, f^{(2)}, \cdots f^{(n)}$ is the shape of a monotone gapless triangle if and only if for any $k<n$ the words $f^{(k)}$ and $f^{(k+1)}$ satisfy the condition given above for $g$ and $h$.

Proof. It suffices to remark that the $a_{i, j}$ of the corresponding monotone triangles are obtained from the $f^{(i)}$ by the relation

$$
a_{i, j}=j+\sum_{k=1}^{n-i} f_{k}^{(n-j)}
$$

so that the above inequality is a reformulation of the relation $a_{i, j}<a_{i, j+1}$. 


\section{Growth processes for gapless shapes}

In the sequel gapless shape will mean shape of a gapless monotone triangle. That is a triangle $s_{i j}$ with entries 0 and 1 whose columns satisfy the condition in Proposition 1.

\subsection{Column insertion}

A natural way to build a gapless shape is to proceed in giving the words $f^{(1)}, f^{(2)}, \cdots, f^{(n)}$ successively. The choice of possible $f^{(n)}$ depends only on the value of $f^{(n-1)}$. Moreover all the $f^{(n)}$ which may follow a given $f^{(n-1)}$ can be built by using a simple process which we describe below.

For that purpose we consider some words on the alphabet $\{a, b\}$. We recall that a word $w$ on this alphabet is a Dyck word if for any prefix $w^{\prime}$ of $w$ the number of occurrences of the letter $a$ (denoted $\left|w^{\prime}\right|_{a}$ ) is not less than the number of occurrences of the letter $b$ (denoted $\left|w^{\prime}\right|_{b}$ ). We now introduce a mapping $\phi$ associating to two words of length $n-1$ and $n$ on the alphabet $\{0,1\}$ a word of length $2 n-1$ on the alphabet $\{a, b\}$.

We give first a mapping $\theta$ giving for each pair of letters in $\{0,1\}$ a word of length 2 on the alphabet $\{a, b\}$ :

$$
\theta(0,0)=a b, \quad \theta(1,1)=b a, \quad \theta(1,0)=a a, \quad \theta(0,1)=b b .
$$

Considering two words $g$ and $h$ on the alphabet $\{0,1\}$ of lengths $|h|=|g|+1=n$ we associate a word $w=\phi(g, h)$ of length $2 n-1$ on the alphabet $\{a, b\}$ given by

$$
w=a \theta\left(g_{1}, h_{1}\right) \theta\left(g_{2}, h_{2}\right) \cdots \theta\left(g_{n-1}, h_{n-1}\right) .
$$

For example, the word corresponding to the first two columns in the shape (3.2), $g=(1,1)$ and $h=(1,0,1)$, is

$$
w=\phi(g, h)=a \theta(1,1) \theta(1,0)=a b a a a,
$$

which is a valid Dyck word.

Proposition 2. Two words $g$ and $h$ of lengths $n-1$ and $n$ respectively correspond to two consecutive columns in a gapless shape if and only if the word $w=\phi(g, h)$, is a prefix of a Dyck word.

Proof. The condition on the partials sums of $g$ and $h$ given in Proposition 1 translates naturally into counting the number of occurrences of $a$ and $b$ in $\phi(g, h)$.

Corollary 1. The number of pairs of words $g, h$ such that

$$
|h|=|g|+1=n
$$

and $g$ and $h$ represent consecutive columns in a gapless shape is equal to the central binomial coefficient

$$
\left(\begin{array}{c}
2 n \\
n
\end{array}\right)
$$


Proof. Using the well-known fact that the number of prefixes of Dyck words of length $2 n-1$ is equal to the binomial coefficient $\left(\begin{array}{c}2 n-1 \\ n\end{array}\right)$, and that the last letter of $h$ may be 0 or 1 , we get $2\left(\begin{array}{c}2 n-1 \\ n\end{array}\right)=\left(\begin{array}{c}2 n \\ n\end{array}\right)$.

We used the column insertion construction to compute the number of all gapless shapes of monotone triangles up to size 12 obtaining the numbers below. For comparison, we also list the total number of ASMs.

\begin{tabular}{|c|c|c|}
\hline Size & Number of ASMs in bijection & Total number of ASMs \\
\hline 1 & 1 & 1 \\
2 & 2 & 2 \\
3 & 6 & 7 \\
4 & 26 & 42 \\
5 & 162 & 429 \\
6 & 1450 & 7436 \\
7 & 18626 & 218348 \\
8 & 343210 & 10850216 \\
9 & 9069306 & 911835460 \\
10 & 343611106 & 129534272700 \\
11 & 18662952122 & 31095744852375 \\
12 & 1453016097506 & \\
\hline
\end{tabular}

Table 1: The number of ASMs in bijection and the total number of ASMs.

\section{$5 \quad$ Rectangular shapes}

Although it seems difficult to obtain a closed formula for the number of triangular shapes, the number of rectangular ones have a nice enumeration formula.

Definition 3. A gapless rectangular shape is a rectangular array $r_{i, j}$ where $1 \leq i \leq p$, $1 \leq j \leq m$ of 0 's and 1's such that for any $j, i$ (where $1 \leq j<m$ and $1 \leq i \leq p$ ),

$$
\sum_{k=i}^{p} r_{k, j} \leq \sum_{k=i}^{p} r_{k, j+1}
$$

To any rectangular shape can be associated a cumulant rectangular array $s_{i, j}$ such that $s_{i, j}=\sum_{k=i}^{p} r_{k, j}$. Then the condition on $r$ translates on $s$ into

$$
s_{i, j} \leq s_{i, j+1} .
$$

Moreover since $r_{i, j} \in\{0,1\}$,

$$
s_{i, j+1}-1 \leq s_{i, j} \leq p-i+1 .
$$

An example of a gapless rectangle and its cumulant array is given in Figure 1. 


$\begin{array}{llllllllllllllllll}0 & 0 & 0 & 0 & 1 & 1 & 1 & 1 & 0 & 0 & 1 & 1 & 1 & 3 & 3 & 3 & 3 & 3 \\ 0 & 0 & 0 & 0 & 1 & 1 & 1 & 0 & 1 & 0 & 1 & 1 & 1 & 2 & 2 & 2 & 2 & 3 \\ 0 & 1 & 1 & 1 & 1 & 0 & 0 & 1 & 1 & 0 & 1 & 1 & 1 & 1 & 1 & 1 & 2 & 2 \\ 0 & 0 & 0 & 0 & 0 & 1 & 1 & 1 & 1 & 0 & 0 & 0 & 0 & 0 & 1 & 1 & 1 & 1\end{array}$

Figure 1: A gapless rectangle and its cumulant array

To any cumulant array is associated a triangular array $t_{i, j}$ such that $t_{i, j}$ is the smallest $k$ such that $s_{n+2-i-j, k} \geq j$ if such $k$ exists and equal to $m+1$ otherwise. The triangular array associated to the cumulant array in Figure 1 is given by

$\begin{array}{llll}6 & 8 & 9 & 10 \\ 2 & 5 & 5 & \\ 2 & 5 & & \\ 2 & & & \end{array}$

It turns out that the triangular array built above belongs to a family of arrays called branchings and considered in Gel72] and in [CS75.

Definition 4. . A p-branching is a triangular array of positive integers $b_{i, j}$ where $1 \leq$ $i \leq p, 1 \leq j \leq p+1-i$ such that the entries are weakly increasing along rows and weakly decreasing along columns,

$$
b_{i+1, j} \leq b_{i, j} \leq b_{i, j+1}
$$

The relation between gapless rectangular shapes, cumulant arrays and $p$-branchings may be summarized in the following statement.

Proposition 3. The above construction is a bijection between gapless rectangles of size $p, m$ and $p$-branchings with entries less or equal to $m+1$.

Recall that a semi-standard Young tableau is an array $t_{i, j}$ of entries weakly increasing along rows, strictly increasing along columns, that is

$$
t_{i, j} \leq t_{i, j+1} \text { and } t_{i, j}<t_{i+1, j} .
$$

Then the following proposition allows us to obtain an enumeration formula for gapless rectangles.

Proposition 4. Let $p, m$ be two integers not less than 1. There is a bijection between p-branchings with entries not greater than $m$ and the set of semi-standard Young tableaux with entries in $1,2, \ldots p$ and having less than $m$ columns.

Proof. Consider the branching $B=\left(b_{i, j}\right)$, we build a semi-standard Young tableau $T$ associated to it performing the following algorithm:

For each $i$ the $i$-the row of $T$ has length $k_{i}-1$ where $k_{i}$ is the largest value appearing in the $i$ th row of $B$. Then $t_{i, j}$ is equal to $p+1$ minus the number of elements in the $i$-th row of $B$ which are not less than $j+1$. The fact that $T$ is indeed a semi-standard Young tableau follows from two simple remarks. The first one shows that $t_{i, j} \leq t_{i, j+1}$ and the second shows that $t_{i, j}<t_{i+1, j}$. 
- Let $u=u_{1}, u_{2}, \ldots, u_{i}$ be any sequence of integers and let $a_{k}$ be the number of elements in $u$ not less than a given integer $k$ then $a_{k} \geq a_{k+1}$.

- Let $u=u_{1}, u_{2}, \ldots u_{i}$ and $v=v_{1}, v_{2}, \ldots, v_{i-1}$ be two non decreasing sequences of integers such that for all $j<i, u_{j} \geq v_{j}$ and let $a_{k}$ (resp. $b_{k}$ ) be the number of elements in $u$ (resp. $v$ ) not less than a given integer $k$. Then $a_{k}>b_{k}$ for all $k$.

Conversely given $p, m$ and a semi-standard Young tableau $T$ with entries not greater than $p$ and having at most $m$ columns we build the branching $B$ by the process described below.

For each $i$ from 1 to $p$, the $i$-th row of $B$ (which has length $p+1-i$ ) consists of $t_{i, k}-t_{i, k-1}$ entries equal to $k$ for each $k>1$. If the length of the $i$ th row of $T$ is $l_{i}$, fill in $p+1-t_{i, l_{i}}$ entries equal to $l_{i}+1$. Since the $i$ th row of $B$ has to have length $p+1-i$, we fill the row with remaining entries equal to 1 and arrange the entries in non-decreasing order.

To illustrate the above bijection we give the semi-standard Young tableau associated to the branching in (5.2),

$\begin{array}{lllllllll}1 & 1 & 1 & 1 & 1 & 2 & 2 & 3 & 4 \\ 2 & 3 & 3 & 3 & & & & & \\ 3 & 4 & 4 & 4 & & & & & \\ 4 . & & & & & & & & \end{array}$

Corollary 2. The number $\rho_{m, p}$ of rectangular gapless shapes with $p$ rows and $m$ columns is equal to,

$$
\rho_{m, p}=\prod_{i=1}^{p} \frac{\left(\begin{array}{c}
m+2 i-1 \\
i
\end{array}\right)}{\left(\begin{array}{c}
2 i-1 \\
i
\end{array}\right)}=\prod_{i=1}^{p} \prod_{j=i}^{p} \frac{m+i+j-1}{i+j-1} .
$$

Proof. The number of such rectangles is by the above bijections equal to the number of semi-standard Young tableaux with entries in $1,2, \ldots, p$ and having less than $m+1$ columns, which is also the number of column-strict partitions (see [Sta71]).

\subsection{Decomposition of a triangular gapless shape}

For any integer $k \leq n$ triangular gapless shape of size $n$ can be decomposed into a rectangle with $k$ rows (and $n-k$ columns) and two triangles of sizes $k$ and $n-k$ as shown in Figure 2

From this decomposition we get the following asymptotic estimate on the number of gapless triangular shapes.

Proposition 5. The number $a_{n}$ of gapless triangular shapes of size $n$ satisfies the inequality:

$$
a_{n} \geq \rho_{n-k, k} a_{n-k}
$$

where $\rho_{m, k}$ denotes the number of rectangular gapless shapes with $k$ rows and $m$ columns. 


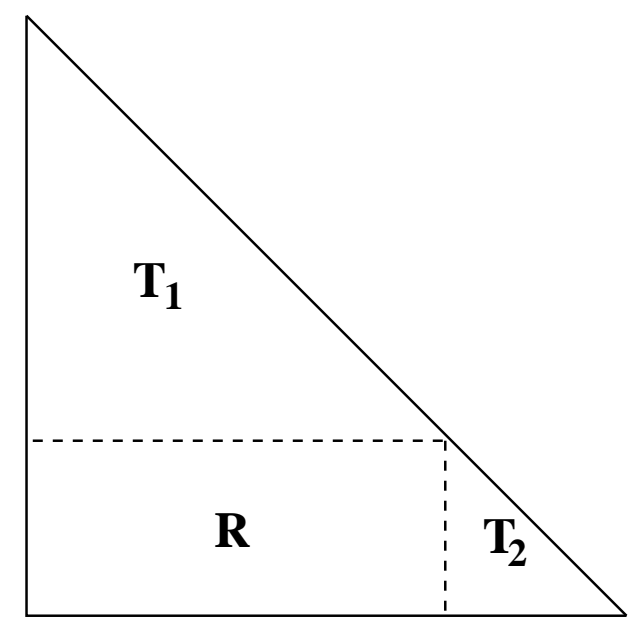

Figure 2: Decomposition of a gapless triangle

Proof. Given a rectangular gapless shape $\mathbf{R}$ with $k$ rows and $n-k$ columns, we build a triangular gapless shape $T$ adding above any triangular shape $T_{1}$ and on its right a triangular shape $T_{2}$ in which all entries are equal to 1.

A lower bound for an asymptotic evaluation purpose may be obtained when taking $k=\frac{n}{2}$.

Corollary 3. Consider the sequence of integers given by

$$
\alpha_{1}=1, \alpha_{2}=2, \alpha_{3}=6, \alpha_{4}=26
$$

and

$$
\alpha_{n}=\rho_{\left\lceil\frac{n}{2}\right\rceil,\left\lfloor\frac{n}{2}\right\rfloor} \alpha_{\left\lceil\frac{n}{2}\right\rceil} .
$$

Then the number of gapless shapes of size $n$ is greater than $\alpha_{n}$.

However, numerics suggest that the true estimate is

$$
a_{n} \sim\left(\frac{3^{9}}{2^{12}}\right)^{\frac{n^{2}}{8}}
$$

It would be interesting to derive this from a formula for $a_{n}$.

\section{Magog Triangles}

We now prove a bijection between Magog triangles (representing totally symmetric selfcomplementary plane partitions) which avoid a certain pattern and 312 avoiding Gog words. 
Definition 5. A Magog triangle or fundamental domain for partitions of size $n$ is an array $\left(b_{i j}\right)$ of positive integers defined for $n \geq i \geq j \geq 1$ that is written in the form

$$
\begin{array}{cccc}
b_{1,1} & & & \\
b_{2,1} & b_{2,2} & & \\
\vdots & \vdots & \ddots & \\
b_{n, 1} & b_{n, 2} & \cdots & b_{n, n},
\end{array}
$$

where,

1. $b_{i, j} \leq n$,

2. $b_{i, j} \leq b_{i+1, j}$ and $b_{i, j} \leq b_{i, j+1}$ whenever both sides are defined (weakly decreasing along both directions),

3. $b_{i, j} \geq i$.

Definition 6. A Magog triangle $b$ contains a gap at position $(i, j)$ if $b_{i+1, j}-b_{i, j}>1$. A gapless Magog triangle is a Magog triangle which contains no gaps.

Notice that the above condition is very similar to that defining gapless monotone triangles in Definition 2. We showed in Section [ that Gog words which avoid the generalized 312 pattern are in bijection with gapless monotone triangles, but we have not been able to find an interpretation for gapless Magog triangles. Since Magog triangles were motivated by and are in bijection with totally symmetric self-complementary plane partitions, one could hope for a natural interpretation in that context.

Consider the following transformation $\Delta$ associating to any monotone triangle $\left(a_{i j}\right)$ with integer coefficients a new triangle $\left(b_{i j}\right)$ given by

$$
b_{i, j}=a_{i, j}+i-j
$$

Theorem 4. For a monotone triangle $T=\left(a_{i j}\right)$ the triangle $\Delta(T)$ is a Magog triangle if and only if $T$ is gapless. Furthermore, $\Delta(T)$ is a gapless Magog triangle.

Proof. The proof is straightforward and left to the reader.

We give an example to illustrate the idea. The gapless monotone triangle,

$$
\begin{array}{llll}
2 & & & \\
2 & 3 & & \\
1 & 2 & 4 & \\
1 & 2 & 3 & 4,
\end{array}
$$

maps to the gapless Magog triangle,

$$
\begin{array}{llll}
2 & & & \\
3 & 3 & & \\
3 & 3 & 4 & \\
4 & 4 & 4 & 4 .
\end{array}
$$


Notice that whenever the difference between the entries at positions $(i, j)$ and $(i+1, j)$ in the gapless monotone triangle was $\delta(\delta=0,1$ by definition), the difference between the entries at positions $(i+1, j)$ and $(i, j)$ in the Magog triangle was $1-\delta$. This is an obvious consequence of the bijection.

\section{$7 \quad 312$ patterns for permutations}

We will now show that a permutation avoids the pattern 312 if and only if the monotone triangle associated to it is gapless. First, we remind the reader of the definition of pattern avoidance for the specific case of the pattern 312 .

Definition 7. A permutation $\pi$ of the letters $\{1, \ldots, n\}$ contains the pattern 312 if there exist positive integers $i, j, k$, with $1 \leq i<j<k \leq n$ such that $\pi_{i}>\pi_{k}>\pi_{j}$. If no such triple of integers exists, the permutation $\pi$ is said to avoid the pattern 312 .

Before we demonstrate the bijection, we need a simple but useful observation. This correspondence has been noted before (Lemma 2 in [Cla01, but we repeat it for the sake of convenience.

Remark 2. If a permutation $\pi$ contains the pattern 312 at positions $(i, j, k)$ and $j-i>1$, then there exists another integer $l, i \leq l \leq j$ such that the permutation contains another pattern 312 at $(l, l+1, k)$. The proof of this comes by looking at the integer $\pi_{i+1}$. If $\pi_{i+1}<\pi_{k}$ then $(i, i+1, k)$ is the required triple. If not, then $(i+1, j, k)$ is the new triple and we iterate.

Another way of saying this is that permutations containing or avoiding 312 are also those containing or avoiding 31-2 respectively. For example, the permutation $\pi=\underline{5} 42 \underline{1} 6 \underline{3}$ has a 312 pattern as shown. The iteration is as follows,

$$
\underline{5} 42 \underline{1} 6 \underline{3} \rightarrow 5 \underline{4} 2 \underline{1} 6 \underline{3} \rightarrow 5 \underline{42} 16 \underline{3} .
$$

We use the above observation to refine the definition of containment of the 312 pattern.

Definition 8. We say that a permutation $\pi$ contains the 312 pattern at position $i$ if $\pi$ contains a 312 pattern at positions $(i, i+1, k)$ for some $k>i+1$.

Remark 2 leads to the following proposition.

Lemma 1. Assuming that a permutation $\pi=\left(\pi_{1}, \ldots, \pi_{n}\right)$ does not contain a 312 pattern at any position less than $i$, it avoids the 312 pattern at position $i$ if and only if the set $S_{i}=\left\{\pi_{j} \mid j \leq i+1, \pi_{j} \geq \pi_{i+1}\right\}$ consists of consecutive integers.

Proof. Suppose there are no 312 patterns at positions less than $i$. The only way to get a 312 pattern at position $i$ is to have $\pi_{i}>\pi_{i+1}+1$ and have a $k$ such that if $\pi_{i+1}<\pi_{k}<\pi_{i}$, then $k>i+1$. But if $S_{i}$ is consecutive, all such $k$ 's will necessarily satisfy $k<i$. 
For the converse, assume that $S_{i}$ is not consecutive. Therefore, there exists a smallest $k$ such that $\pi_{k}>\pi_{i+1}$ and $\pi_{k} \notin S_{i}$. Now, if $\pi_{i}<\pi_{k}$, then $S_{i-1}$ is not consecutive either, which is a contradiction.

Therefore, we must have $\pi_{i}>\pi_{k}$, in which case $\left(\pi_{i}, \pi_{i+1}, \pi_{k}\right)$ is the required 312 pattern.

As a consequence of Lemma 1, we need to only look at the $(i+1)$ 'th prefix of a permutation to see if it contains a 312 pattern at a position less than $i$. Of course, to ensure that the permutation avoids 312 , we must look at the entire permutation.

We now see what 312 containment in a permutation means for the corresponding monotone triangle. See the paragraph above (2.2) for an explanation of this correspondence.

Lemma 2. A permutation $\pi$ on $n$ letters contains the pattern 312 at position $i$ (assuming no 312 pattern at positions less than i) if and only if there exists a gap in the corresponding monotone triangle a at row $i$.

Proof. Consider rows $i, i+1$ for the monotone triangle $a$. Suppose $a_{i+1, j}=\pi_{i+1}$ and look at columns starting at column $j$ because the columns from 1 to $j-1$ will be unchanged. Since $\pi_{i+1}$ is the only new entry in row $i+1$, all the entries in row $i$ larger than it will be shifted to the right by one in column $i+1$ as shown,

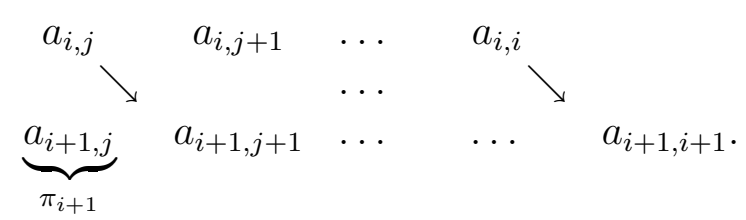

Notice that the set $\left\{a_{i+1, j}, \ldots, a_{i+1, i+1}\right\}$ equals $S_{i}$. As a consequence of Lemma 11, it is clear that there will be no gaps if and only if this set is consecutive.

Remark 3. If a permutation contains the pattern 312 at position $i$, then there could be multiple columns where the gap occurs at row $i$. For example, the permutation $3 \underline{514} 2$ contains a 312 at the second position but the monotone triangle

$\begin{array}{lllll}3 & & & & \\ \underline{3} & \underline{5} & & & \\ 1 & 3 & 5 & & \\ 1 & 3 & 4 & 5 & \\ 1 & 2 & 3 & 4 & 5,\end{array}$

contains two gaps in the second row.

The number of gaps in a monotone triangle corresponding to a given permutation containing 312 is exactly the number of 31-2 patterns. An exact formula for $\phi_{k}(n)$, the number of permutations of size $n$ with $k$ 31-2 patterns, is not known. Parviainen Par06 has given an algorithm for computing this and exact formulas for the number up to $k=8$. 


\section{Gog words}

We now define words which are enumerated by the ASM numbers and are in natural bijection with alternating sign matrices. Before that, we need an alphabet, the set of which itself depends on $n$.

Definition 9. The Gog alphabet, $\mathcal{A}$, is given by tuples $\left(p_{1}, q_{1}, \ldots, p_{k-1}, q_{k-1}, p_{k}\right)$ such that each $1 \leq p_{1}<q_{1} \ldots p_{k-1}<q_{k-1}<p_{k}$.

For example, $\mathcal{A}$ contains all the integers but in addition contains elements such as $(1,2,3)$ and $(7,9,10,13,17)$. In all examples, we will avoid commas between the entries in a tuple throughout the article.

Definition 10. A Gog word $w$ of size $n$ is a word consisting of elements of $\mathcal{A}$ satisfying the following conditions:

1. The length of the word is $n$,

2. Each letter in the word has maximum entry at most n,

3. An integer in an even numbered position in a tuple is repeated in another tuple to its left and to its right in odd numbered positions,

4. Every repeated integer alternates in odd and even numbered positions in subsequent tuples.

Notice that the first and last letter has to be of size one. Clearly, Gog words with alphabets of size one correspond to permutations. We omit the brackets around singleton elements (i.e., we write 2 instead of (2)) for ease of reading. For example 2(123)2 and 213 are both Gog words of size 3 and 25(12456)(345)(234)3 is a Gog word of size $n=6$.

A word about notation. We will denote elements of the alphabet $\mathcal{A}$, also called tuples, by $x_{i}$, the lengths of these elements by $2 k_{i}-1$, odd elements of these tuples by $p_{j}$ and even elements by $q_{j}$. Elements of the monotone triangle will be denoted using the same subscripts, e.g. $a_{i, j}$.

Remark 4. There is a natural bijection relating Gog words and alternating sign matrices. The jth tuple of a Gog word is simply the positions, in increasing order, of nonzero entries in the jth row in the corresponding alternating sign matrix. The even numbered positions correspond to -1 and the odd numbered ones, to +1 .

Remark 5. There is a natural bijection relating Gog words and monotone triangles. Given a Gog word $x_{1} \ldots x_{n}$, the first row of the corresponding monotone triangle is simply the first element and the ith row is obtained by taking the $i-1$ th row, removing the evenpositioned elements in $x_{i}$ from it, appending the odd-positioned elements in $x_{i}$ to it, and sorting in increasing order.

THE ELECTRONIC JOURNAL OF COMBINATORICS 18(2) (2011), \#P26 
Given a monotone triangle a, the first tuple is the singleton element in the first row, and the ith tuple in the corresponding Gog word is given by listing the sequence

$$
\left(a_{i, 1}, a_{i-1,1}, \ldots, a_{i, i-1}, a_{i-1, i-1}, a_{i, i}\right)
$$

and removing occurrences of every repeated integer.

For an example, consider the monotone triangle,

$\begin{array}{llll}3 & & & \\ 1 & 3 & & \\ 1 & 2 & 4 & \\ 1 & 2 & 3 & 4 .\end{array}$

We construct first the lists of sequences (3)(133)(11234)(1122344) and then remove every occurrence of repeated integers to get $(3)(1)(234)(3)$. Both the bijections in Remark 4 and Remark 5 are commensurate with the well-known bijection relating monotone triangles and ASMs MRR83.

Notice that a Gog word is not a permutation of letters in the alphabet $\mathcal{A}$, as the example 2(123)2 shows.

\section{2 subpatterns for Gog words}

We showed in Lemma 2 that a 312 pattern in a permutation is equivalent to a column-wise difference greater than one in the corresponding monotone triangle. A natural generalization would be to interpret the occurrence of this column-wise difference in an arbitrary monotone triangle as the occurrence of a generalized 312 pattern in the corresponding Gog words.

For example, the monotone triangle in (8.2) corresponds to the word 31(234)3 and the fact that the $(1,1)$ th entry minus the $(1,2)$ th entry is greater than one can be interpreted as an occurrence of a generalized 312 pattern. We now make this notion precise.

Definition 11. We say that an integer $m$ is active with respect to a tuple $x=\left(p_{1}, q_{1}, \ldots\right.$, $\left.p_{k-1}, q_{k-1}, p_{k}\right)$ if $m>p_{k}$ or if $p_{j}<m<q_{j}$ for $j \in[k-1]$.

Definition 12. We say that integers $c, a, b$ form a 312-subpattern of the Gog word $w=$ $x_{1} x_{2} \ldots x_{n}$ if the following conditions hold

1. $c, a, b$ appear in odd positions in $x_{i}, x_{j}, x_{k}$ respectively where $i<j<k$,

2. $b$ is not in an even position in $x_{i+1}, \ldots, x_{k-1}$,

3. $b$ is active with respect to $x_{j}$.

4. $a<b<c$.

THE ELECTRONIC JOURNAL OF COMBINATORICS 18(2) (2011), \#P26 
In the above example (8.2), $\underline{31}(\underline{2} 34) 3$ contains the 312 -subpattern. The following two examples illustrate the subtlety of the activity condition.

$$
2 \underline{5}(\underline{12} 356) 5 \underline{4} 2
$$

contains a 312-subpattern but

$$
2 \underline{5}(\underline{1} 2456) 5 \underline{3} 2
$$

does not.

This definition is stronger than the one used in [JL07, whose conditions, in this language, amount to (1) and (4) in Definition 12. Therefore, the number of Gog words avoiding the 312-subpattern is bounded below by the number of alternating sign matrices avoiding the permutation matrix of the permutation 312 .

Remark 6. If a Gog word $w=x_{1} \ldots x_{n}$ contains the 312-subpattern $(c, a, b)$ at positions $x_{i}, x_{j}, x_{k}$ whose lengths are $2 p_{i}-1,2 p_{j}-1$ and $2 p_{k}-1$ respectively, then $\left(\left(x_{i}\right)_{2 p_{i}-1},\left(x_{j}\right)_{1}, b\right)$ is also a 312-subpattern since $\left(x_{i}\right)_{2 p_{i}-1} \geq c$ and $\left(x_{j}\right)_{1} \leq a$. Notice that the activity condition is unaffected.

A natural question would be whether Remark 2 generalizes to Gog words. The following lemma shows that it does.

Lemma 3. If a Gog word $w=x_{1} \ldots x_{n}$ contains the 312-subpattern, then there exist positions $i, k$ such that the subpattern $(c, a, b)$ appears in $x_{i}, x_{i+1}, x_{k}$.

Proof. The idea of the proof is very similar to that in Remark 2. Suppose that the 312subpattern in $w$ occurs at $x_{i}, x_{j}, x_{k}$ respectively. It follows from Remark 6 that we can take $c=\left(x_{i}\right)_{2 p_{i}-1}$ and $a=\left(x_{j}\right)_{1}$. Now look at the last entry in $x_{i+1}, p=\left(x_{i+1}\right)_{2 p_{i+1}-1}$.

First, note that $p$ cannot be equal to $b$ because $b$ is present in an odd position in $x_{k}$ and therefore, that would force $b$ to be in an even position between $x_{i+1}$ and $x_{k}$, which is forbidden in the definition of the 312-subpattern.

If $p>b$, then $(p, a, b)$ is a 312-subpattern and we iterate. If $p<b$, then $(c, p, b)$ is a possible 312-subpattern if we can show that $b$ is active with respect to $x_{i+1}$. But this is guaranteed since $b$ is larger than $p$, which is the largest element in $x_{i+1}$. This proves the lemma.

We will now generalize Lemma 1 from permutations to Gog words.

Lemma 4. Let $w=x_{1} \ldots x_{n}$ be a Gog word and a be the corresponding monotone triangle. Let $x_{i+1}=\left(p_{1}, q_{1}, \ldots, p_{k-1}, q_{k-1}, p_{k}\right)$. Define

$$
\begin{aligned}
& P_{l}=\left\{a_{i, j} \mid q_{l} \geq a_{i, j}>p_{l}\right\}, \quad \text { for } l<k \text { and } \\
& P_{k}=\left\{a_{i, j} \mid a_{i, j} \geq p_{k}\right\} .
\end{aligned}
$$

Then there is no gap at row $i$ in $a$ if and only if $P_{l} \cup\left\{p_{l}\right\}$ for $l<k$ and $P_{k}$ consist of consecutive integers. 
Proof. We begin with an innocuous observation. Let $w=x_{1} \ldots x_{n}$ be a Gog word of size $n$ and $a$ be the corresponding monotone triangle. Let $x_{j}=\left(p_{1}, q_{1}, \ldots, p_{k-1}, q_{k-1}, p_{k}\right)$. For any $i$, if $p_{i}=a_{i, r}, q_{i}=a_{i-1, s}$ and $p_{i+1}=a_{i, t}$, then $r \leq s<t$.

This is simply a consequence of the definition of a monotone triangle. Suppose $s<r$, and part of the monotone triangle looks like

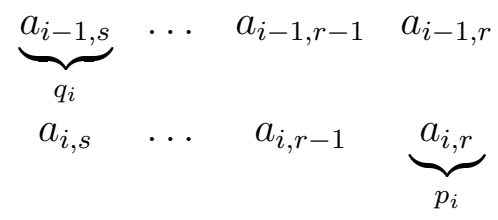

Then $q_{i} \leq a_{i-1, r-1} \leq p_{i}$, which is a contradiction. Similarly, suppose $t \leq s$, from which part of the monotone triangle looks like

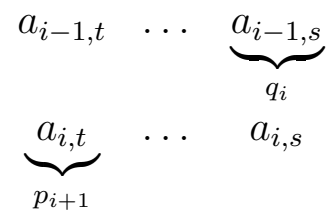

which implied $p_{i+1} \leq a_{i-1, t} \leq q_{i}$, which is again a contradiction.

From this it follows that the set of elements $\left\{a_{i-1, j} \mid r \leq j<s\right\}$ is exactly the same as the set $P_{i}$,

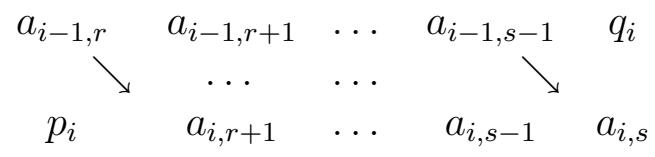

because none of the elements in $P_{i}$ is removed. It is now clear that if the elements in $P_{i}$ are consecutive, then there cannot be a gap between columns $r$ and $s$. This argument works for all $i<k$. For $i=k$, the argument is identical to that in the proof of Lemma 2,

We are now in a position to generalize Lemma 2 to Gog words.

Theorem 5. Assuming that a Gog word $w$ of size $n$ does not contain the 312-subpattern at positions less than $i$, it contains the 312-subpattern at position $i$ if and only if there exists a gap in the corresponding monotone triangle a at row $i$.

Proof. Combining what we have already shown, it remains to show, using the notation of Lemma 4 that the existence of a 312 pattern of row $i$ is equivalent to the existence of a $P_{l}$ which is not consecutive.

Suppose the 312-subpattern is obtained by the triple of integers $(c, a, b)$. We first show that $b$ cannot be any of the $p_{l}$ 's or $q_{l}$ 's. Clearly $b$ cannot be equal to any $q_{l}$ because then it is in an even position in $x_{i+1}$, which is forbidden by definition. $b$ cannot also be equal to any $p_{l}$ because it will be forced to be in an even position strictly between $x_{i+1}$ and $x_{k}$ as a consequence of being a Gog word, which is forbidden for the same reason.

If $p_{k}>b, P_{k}$ is not consecutive and we are done. Otherwise, by the activity condition, there exists $l$ such that $p_{l} \leq b \leq q_{l}$ and then $P_{l}$ is not consecutive. As a consequence of Lemma 4, we have a gap at row $i$. 
For the converse, suppose there is a gap at row $i$. By Lemma 4, either $P_{l}$ for $l<k$ or $P_{k}$ is not consecutive. In either case, there exists an integer $x$ in row $i$ and an integer $z$ in row $i+1$ in the same column as $x$ such that $x-z>1$. Choose an integer $b$ such that $x<b<z$. By choice, $b$ is active with respect to $x_{i+1}$. Let $x_{m}$ be the first tuple in which $b$ appears in an odd position after $x_{i+1}$. By construction, $b$ is not in an even position in tuples between $x_{i+1}$ and $x_{m}$. We set $a=p_{1}$ and $c$ to be the largest integer in $x_{i}$. Clearly $a<b$ as $a<x$.

All that remains to be done is to prove $b<c$. We will show that by demonstrating that the column in which $c$ belongs cannot be to the left of that of $x$ and $z$. If that happens, rows $i-1, i$ and $i+1$ will look as follows,

$$
\begin{array}{lllll}
* & \ldots & x & * & \ldots \\
c & \ldots & z & x & \ldots \\
* & \ldots & * & z & \ldots,
\end{array}
$$

and as shown, there will be a gap between rows $i-1$ and $i$ by the same $x$ and $z$, which is forbidden by assumption. Therefore $c$ cannot be in a column to the left of that of $x$ and $z$. It can, of course, be in the same column (i.e. $x=c$ ), or to the right. In both cases $b<c$ and thus, $(c, a, b)$ is the required 312 pattern.

\section{Conclusions}

We have defined a new set of words on an infinite alphabet which are equinumerous with alternating sign matrices. Further, we showed that there is a notion of 312-avoidance which has a natural interpretation in terms of both monotone triangles (column-wise difference less than two) and in terms of ASMs (natural bijection with TSSCPPs). However, a number of questions remain unanswered.

First, we would like to understand better how the number of monotone triangles in bijection with the subset of magogs grows with the size. We have shown that ASMs grow superexponentially in size (B.7) (see also [BF06]), with entropy $\lambda_{1} \approx 1.29904$ (B.8). We have been able to show that the number of ASMs in bijection grow like (A.17), with entropy $\lambda_{2} \approx 1.13975(\mathrm{~B} .9)$. For some strange reason, we find that $\lambda_{1}=\lambda_{2}^{2}$. What we conjecture is that the entropy is actually, using (5.3), given by $\frac{3^{9 / 8}}{2^{3 / 2}} \approx 1.21679$.

Secondly, we observe that we do not know if there is a meaningful interpretation of this bijection on the TSSCPP side. If one believes that this bijection is not artificial, then one would expect some structure on TSSCPPs. We have not been able to find this structure so far.

Finally, one would be interested in exploiting other pattern avoidances on Gog words. The definition of 312-subpatterns uses a notion of activity that seems somehow specific to the subpattern 312. One would like a more holistic definition, which encompasses other subpatterns. 


\section{Acknowledgements}

We thanks P. Duchon, O. Guibert and X. Viennot for discussions, Florent Le Gac for collaborating on some of the results in Section 5. J. Propp for informing us about the work of H. Canary, C. Krattenthaler for informing us about the asymptotics of the Barnes G-function and the anonymous referee for a thorough reading of the manuscript.

\section{A Gapless monotone triangles}

A lower bound for the number of gapless monotone triangles is given by the sequence $\alpha_{n}$ defined by:

$$
\alpha_{1}=1, \alpha_{2}=2, \alpha_{3}=6, \alpha_{4}=26, \alpha_{n}=\rho_{\left\lceil\frac{n}{2}\right\rceil,\left\lfloor\frac{n}{2}\right\rfloor} \alpha_{\left\lceil\frac{n}{2}\right\rceil},
$$

where $\rho_{m, p}$ is the number of rectangular gapless shapes with $p$ rows and $m$ columns given by

$$
\rho_{m, p}=\prod_{i=1}^{p-1}\left(\frac{m+i}{i}\right)^{\left\lceil\frac{i}{2}\right\rceil} \prod_{i=1}^{p}\left(\frac{m+2 p-i}{2 p-i}\right)^{\left\lceil\frac{i}{2}\right\rceil}
$$

In order to determine the asymptotic behavior of $\alpha_{n}$ it is convenient to start with the formula giving $\rho_{2 n, 2 n}$ which is

$$
\rho_{2 n, 2 n}=\prod_{i=1}^{2 n-1}\left(\frac{2 n+i}{i}\right)^{\left\lceil\frac{i}{2}\right\rceil} \prod_{i=1}^{2 n}\left(\frac{6 n-i}{4 n-i}\right)^{\left\lceil\frac{i}{2}\right\rceil}
$$

Separating the case $i=2 j$ from $i=2 j-1$ we get:

$$
\begin{aligned}
\rho_{2 n, 2 n}=\prod_{j=1}^{n-1} & \left(\frac{2 n+2 j}{2 j}\right)^{j} \prod_{j=1}^{n}\left(\frac{2 n+2 j-1}{2 j-1}\right)^{j} \\
& \times \prod_{j=1}^{n}\left(\frac{6 n-2 j}{4 n-2 j}\right)^{j} \prod_{j=1}^{n}\left(\frac{6 n-2 j+1}{4 n-2 j+1}\right)^{j}
\end{aligned}
$$

Taking the logarithms we have:

$$
\begin{aligned}
& \log \left(\rho_{2 n, 2 n}\right)=\sum_{1 \leq j<n} j \log (2 n+2 j)-\sum_{1 \leq j<n} j \log (2 j) \\
& +\sum_{1 \leq j \leq n} j(\log (2 n+2 j-1)+\log (6 n-2 j)+\log (6 n-2 j+1)) \\
& -\sum_{1 \leq j \leq n} j(\log (2 j-1)+\log (4 n-2 j)+\log (4 n-2 j+1))
\end{aligned}
$$

Let $f(x)$ be the function

$$
\begin{aligned}
f(x) & =x(\log (2 n+2 x)+\log (2 n+2 x-1)+\log (6 n-2 x) \\
& +\log (6 n-2 x+1))-x(\log (2 x)+\log (2 x-1) \\
& +\log (4 n-2 x)+\log (4 n-2 x+1))
\end{aligned}
$$


which relates to $\rho_{2 n, 2 n}$ by the formula

$$
\log \left(\rho_{2 n, 2 n}\right)=\sum_{j=1}^{n} f(j)-n \log (2)=S_{n}-n \log (2)
$$

The next step consists in approximating the sum $S_{n}=\sum_{j=1}^{n} f(i)$ using the EulerMaclaurin formula,

$$
S_{n}=\frac{f(1)+f(n)}{2}+I_{n}+\sum_{k=2}^{\infty} \frac{B_{k}}{k !}\left(f^{(k-1)}(n)-f^{(k-1)}(1)\right),
$$

where $I_{n}$ is the integral

$$
I_{n}=\int_{1}^{n} f(x) d x
$$

and $B_{k}$ are the Bernoulli numbers $\left(B_{1}=-1 / 2, B_{2}=1 / 6, B_{3}=0, B_{4}=-1 / 30, \cdots\right)$.

We can evaluate $I_{n}$ using the formula:

$$
\int x \log (a x+b) d x=\frac{x^{2}}{2} \log (a x+b)-\frac{x^{2}}{4}-\frac{b x}{2 a}-\frac{b^{2}}{2 a} \log (a x+b),
$$

giving

$$
I_{n}=A(n) n^{2}+B(n) n+C(n),
$$

where

$$
\begin{aligned}
A(n) & =-4 \log (2)-3 \log (n)+\frac{9}{2} \log (6 n-1)-4 \log (4 n+1) \\
& -2 \log (4 n-1)+\frac{9}{2} \log (3 n-1) \\
& +2 \log (2 n+1)-\frac{5}{2} \log (2 n-1)+\frac{1}{2} \log (n+1), \\
B(n) & =\frac{3}{2} \log (6 n-1)-\frac{3}{2} \log (4 n+1)-\frac{1}{2} \log (4 n-1)+\frac{1}{2} \log (2 n+1), \\
C(n) & =-\frac{3}{8} \log (6 n-1)-\frac{1}{8} \log (4 n+1)+\frac{1}{4} \log (4 n-1) \\
& -\frac{1}{2} \log (3 n-1)-\frac{1}{4} \log (2 n+1)+\frac{5}{8} \log (2 n-1)-\frac{1}{2} \log (n+1) .
\end{aligned}
$$

Using the approximation formula

$$
\log (a n+b)=\log (a)+\log (n)+\frac{b}{a n}-\frac{b^{2}}{2 a^{2} n^{2}}+O\left(1 / n^{3}\right),
$$

we obtain the following approximations when $n \rightarrow \infty$

$$
\begin{aligned}
\left.I_{n}+\frac{f(1)+f(n)}{2}-n \log (2)\right) & =n^{2}(9 \log (3)-12 \log (2)) \\
& +n\left(\frac{3}{2} \log (3)-\log (2)\right)+\frac{1}{8} \log (n)+O(1), \\
\frac{B_{2}}{2 !}\left(f^{\prime}(n)-f^{\prime}(1)\right) & =-\frac{1}{6} \log (n)+O(1), \\
f^{(3)}(1)-f^{(3)}(0) & =O(1),
\end{aligned}
$$


giving

$$
\begin{aligned}
\log \left(\rho_{2 n, 2 n}\right)= & n^{2}(9 \log (3)-12 \log (2)) \\
& +n\left(\frac{3}{2} \log (3)-\log (2)\right)-\frac{1}{24} \log (n)+O(1), \\
\rho_{2 n, 2 n}= & \gamma\left(\frac{3^{9 n^{2}+\frac{3}{2} n}}{2^{12 n^{2}+n}}\right) n^{-1 / 24}(1+O(1 / \log (n)))
\end{aligned}
$$

where $\gamma$ is a constant. Hence

$$
\rho_{n, n}=\gamma 2^{-1 / 24}\left(\frac{3^{9 n^{2}+3 n}}{2^{12 n^{2}+2 n}}\right)^{1 / 4} n^{-1 / 24}(1+O(1 / \log (n)))
$$

Since we have

$$
\alpha_{n}=\rho_{n / 2, n / 2} \alpha_{n / 2}
$$

we obtain:

$$
\alpha_{n}=\beta\left(\frac{3^{3 / 4}}{2}\right)^{n^{2}}\left(\frac{3^{3 / 4}}{2^{1 / 2}}\right)^{n} n^{-1 / 24}(1+O(1 / \log (n)))
$$

where $\beta$ is a constant.

\section{B Alternating sign matrices}

Bleher and Fokin BF06 have already given an asymptotic estimate of the number $u_{n}$ of ASMs using Riemann-Hilbert methods. For completeness, we give an estimate using more elementary techniques. Recall that

$$
u_{n}=\prod_{0 \leq i<n} \frac{(3 i+1) !}{(n+i) !}
$$

We proceed as above taking the logarithms,

$$
\begin{aligned}
\log \left(u_{n}\right) & =\sum_{1 \leq i<n}(n-i)(\log (3 i-1)+\log (3 i)+\log (3 i+1)) \\
& -\sum_{1 \leq i<n}(n \log (i+1)+(n-i) \log (n+i))
\end{aligned}
$$

Then we consider the function:

$$
\begin{aligned}
f(x) & =(n-x)(\log (3 x-1)+\log (3 x) \\
& +\log (3 x+1)-\log (n+x))-n \log (x+1),
\end{aligned}
$$

and the integral

$$
I_{n}=\int_{1}^{n-1} f(x) d x
$$


This time we get

$$
\begin{aligned}
I_{n} & =\frac{4}{3}(1-n) \log (2)+\frac{1}{2} n(n-2) \log (3)-n^{2} \log (n) \\
& +\left(\frac{(3 n+2)(3 n-4) \log (3 n-4)+(3 n+4)(3 n-2) \log (3 n-2)}{18}\right) \\
& +\left(\frac{(n-1)(n+1) \log (n-1)}{2}\right. \\
& \left.\frac{+(n+1)(3 n-1) \log (n+1)-(2 n-1)(2 n+1) \log (2 n-1)}{2}\right) .
\end{aligned}
$$

Again the Euler-Maclaurin formula gives

$$
\begin{gathered}
S_{n}-\frac{1}{2}(f(1)+f(n-1))=\frac{f(1)}{2}+f(2) \cdots+f(n-2)+\frac{f(n-1)}{2} \\
=I_{n}+\sum_{k=1}^{\infty} \frac{B_{2 k}}{(2 k) !}\left(f^{(2 k-1)}(n-1)-f^{(2 k-1)}(1)\right) .
\end{gathered}
$$

Using $\log (a n+b)=\log (a)+\log (n)+\frac{b}{a n}-\frac{b^{2}}{2 a^{2} n^{2}}+O\left(1 / n^{3}\right)$, we get

$$
\begin{aligned}
I_{n}+\frac{1}{2}(f(1)+f(n)) & =\frac{1}{9} \log (n)-n\left(2 n+\frac{1}{3}\right) \log (2) \\
& +n\left(\frac{3}{2} n-\frac{1}{2}\right) \log (3)+n+O(1), \\
\frac{B_{2}}{2 !}\left(f^{\prime}(n-1)-f^{\prime}(1)\right) & =-\frac{11}{48} n-\frac{1}{4} \log (n)+O(1), \\
\frac{B_{4}}{4 !}\left(f^{(3)}(n-1)-f^{(3)}(1)\right) & =\frac{299}{23040} n+O(1), \\
\log \left(u_{n}\right) & =-2 n^{2} \log (2)+\frac{3}{2} n^{2} \log (3) \\
& +n \log (\beta)-\frac{5}{36} \log (n)+O(1),
\end{aligned}
$$

which leads to

$$
u_{n}=\gamma\left(\frac{27}{16}\right)^{n^{2} / 2} \beta^{n} n^{-5 / 36}(1+O(1 / \log (n))),
$$

where $\gamma$ and $\beta$ are constants. Notice that the number of ASMs grows like $\left(\lambda_{1}\right)^{n^{2}}$ where

$$
\lambda_{1}=\left(\frac{27}{16}\right)^{1 / 2}=\frac{3 \sqrt{3}}{4},
$$

while we have found the lower bound $\left(\lambda_{2}\right)^{n^{2}}$ for the number of those avoiding 312 is

$$
\lambda_{2}=\frac{3^{3 / 4}}{2}
$$




\section{References}

[BF06] Pavel M. Bleher and Vladimir V. Fokin. Exact solution of the six-vertex model with domain wall boundary conditions. Disordered phase. Comm. Math. Phys., 268(1):223-284, 2006.

[Can08] Hal Canary. Aztec diamonds and Baxter permutations. The Electronic Journal of Combinatorics, 17(1):R105, 2010.

[Cla01] Anders Claesson. Generalized pattern avoidance. European Journal of Combinatorics, 22(7):961 - 971, 2001.

[CS75] L. Carlitz and R. P. Stanley. Branchings and partitions. Proc. Amer. Math. Soc., 55(1):246-249, 1975.

[Gel72] S. Gelbart. Problem 5834. Amer. Math. Monthly, 79:523, 1972.

[JL07] Robert Johansson and Svante Linusson. Pattern avoidance in alternating sign matrices. Ann. Comb., 11(3-4):471-480, 2007.

[MRR82] W. H. Mills, David P. Robbins, and Howard Rumsey, Jr. Proof of the Macdonald conjecture. Invent. Math., 66(1):73-87, 1982.

[MRR83] W. H. Mills, David P. Robbins, and Howard Rumsey, Jr. Alternating sign matrices and descending plane partitions. J. Combin. Theory Ser. A, 34(3):340359, 1983.

[Par06] Robert Parviainen. Lattice path enumeration of permutations with $k$ occurrences of the pattern 2-13. J. Integer Seq., 9(3):Article 06.3.2, 2006.

[Sta71] R. P. Stanley. Theory and application of plane partitions, part 2. Studies in applied Math., 50:259-279, 1971.

[Tem96] Nico M. Temme. Special functions. A Wiley-Interscience Publication. John Wiley \& Sons Inc., New York, 1996. An introduction to the classical functions of mathematical physics.

[Zei96] Doron Zeilberger. Proof of the alternating sign matrix conjecture. Electron. J. Combin., 3(2):R13, 1996. The Foata Festschrift. 